\title{
Instantaneous Phasor Method for Obtaining Instantaneous Balanced Fundamental Components for Power Quality Control and Continuous Diagnostics
}

\author{
John S. Hsu \\ Oak Ridge National Laboratory* \\ Post Office Box 2009, MS 8058 \\ Oak Ridge, Tennessee 37831-8058
}

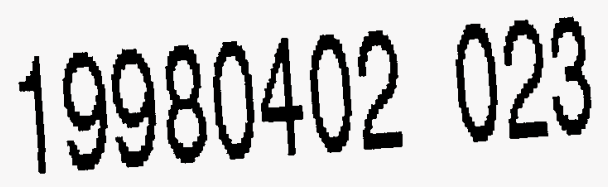

\begin{abstract}
Key words: Method, Instantaneous phasors, Balanced fundamental component, Power quality, Control, Continuous diagnostics.
\end{abstract}

\begin{abstract}
A new instantaneous phasor method for obtaining instantaneous balanced fundamental components is introduced. An example is presented. This technique may be used for active power quality control and for continuous diagnostics.
\end{abstract}

\section{INTRODUCTION}

Poor power quality not only causes additional energy losses, it may also cause detrimental interruptions to business and manufacturing operations. The growing popularity of electronic equipment, such as computers, televisions, electronic ballasts, solid-state motor controllers, and electronically controlled industry loads pollutes the power quality [1]. In 1995, EPRI reported [2] that the revenue losses due to poor power quality to U.S. business alone were $\$ 400$ billion per year.

Poor power quality is caused by unbalanced voltage, under voltage, over voltage, voltage sag, flicker, spike, notch, harmonics, electromagnetic interference, power outage, etc. Power quality requirements are specified in many standards, such as NEMA MG-1 and IEEE-519. For example, the first standard mentioned specifies that voltage unbalance at the motor terminals should not exceed $1 \%$, and the second standard specifies that the total harmonic voltage distortion (THD) at the point of common coupling (PCC) should not exceed $5 \%$ for $69 \mathrm{kV}$ and below. However, in practice the actual voltage unbalance

Paper Number: 98 WM 360.

Accepted for publication in the 1998 IEEE/PES Transactions and harmonics at the power customer's site can far exceed the specified requirements. Often the pollution to the power supply is generated by the load.

The classical power, voltage, and current quality data, such as the reactive power and the root mean square (rms) current and voltage, are quantities evaluated over a period of fundamental cycle. The advent of switching power components, such as the insulated gate bipolar transistors (IGBT), offers advantages for building active power quality improvement devices. The instantaneous power, current, and voltage quality data are essential for control of active power electronic filters and devices.

The term "fundamental" inherently relates to periodic quantities. Therefore, the instantaneous fundamental components correspond to the immediate history of the fundamental components of the past cycle with the adjustment of the present data points. The magnitude and the additional synchronization for severely polluted cases are constantly adjusted.

Initiated by Akagi, Kanazawa, and Nabae, there is a wealth of recent studies on the instantaneous values of reactive power, active current, and reactive current [3-9] that can be used to reduce the reactive component required from the power supply. Willems [6] and Peng et al. [9] further interpreted and developed Akagi-Nabae's power component concept. The instantaneous power is projected to the three-phase voltages to obtain instantaneous active current components. The reactive components of currents can be compensated by power electronics devices even without energy storage [3]. However, if the voltages are unbalanced and contain harmonics, the currents obtained from the power projected onto the voltages wonld alson he

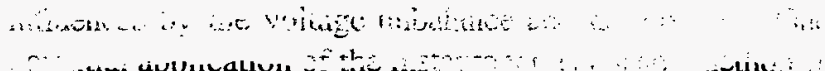
that if the average power can be projected to the fundamental-frequency, balanced voltage components of the supply, the current would contain less harmonics and

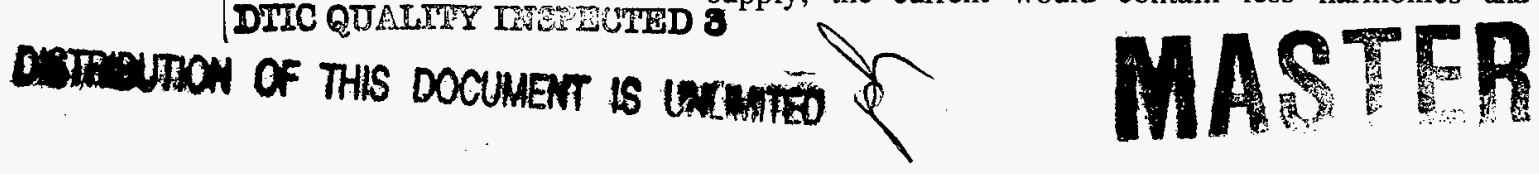




\section{DISCLAIMER}

This report was prepared as an account of work sponsored by an agency of the United States Government. Neither the United States Government nor any agency thereof, nor any of their employees, makes any warranty, express or implied, or assumes any legal liability or responsibility for the accuracy, completeness, or usefulness of any information, apparatus, product, or process disclosed, or represents that its use would not infringe privately owned rights. Reference herein to any specific commercial product, process, or service by trade name, trademark, manufacturer, or otherwise does not necessarily constitute or imply its endorsement, recommendation, or favoring by the United States Government or any agency thereof. The views and opinions of authors expressed herein do not necessarily state or reflect those of the United States Government or any agency thereof. 
unbalance. A limited energy storage may be required to take care of the power fluctuation caused by the unwanted components. In order to do so, the instantaneous fundamental components must be known. Another potential application of this new technology may be in the continuous instantaneous diagnostics area, such as the airgap-torque technology for monitoring efficiency and defects [10-12] of motors.

The immediate history of fundamental balanced components of three-phase voltages or currents that have an equivalent value of three-phase quantities averaged in an rms manner can be obtained by four steps: (1) conducting fast Fourier transform (FFT) of the past-cycle waveforms for each phase individually, (2) shifting phase $b$ and $c$ by 120 and 240 degrees, respectively, in forward and backward directions to find their symmetrical components [13], (3) calculating values for each of the three phases, and (4) computing the equivalent phase value of three-phase quantities averaged in an rms manner. This timeconsuming classical approach is not the method introduced in this paper.

This paper introduces an instantaneous phasor method that considers three phases simultaneously. This method produces the instantaneous fundamental balanced components of the polluted voltages or currents. Fig. 1 shows three-phase voltages that contain $5 \%$ of fundamental magnitude for each order of the 3rd, 5th, 7th, 9th and the 11 th harmonics. respectively. Additionally, the voltages have $5 \%$ unbalance for all voltage components. A $10 \%$ fundamental-frequency zero-sequence component, as well as a $10 \%$ fundamental-frequency negative-sequence component are also added to the phase voltages. Furthermore, certain high-frequency pulses arbitrarily given at $5 \%$ of a 35 th order to represent a possible carrier frequency of power electronic circuits are also included.

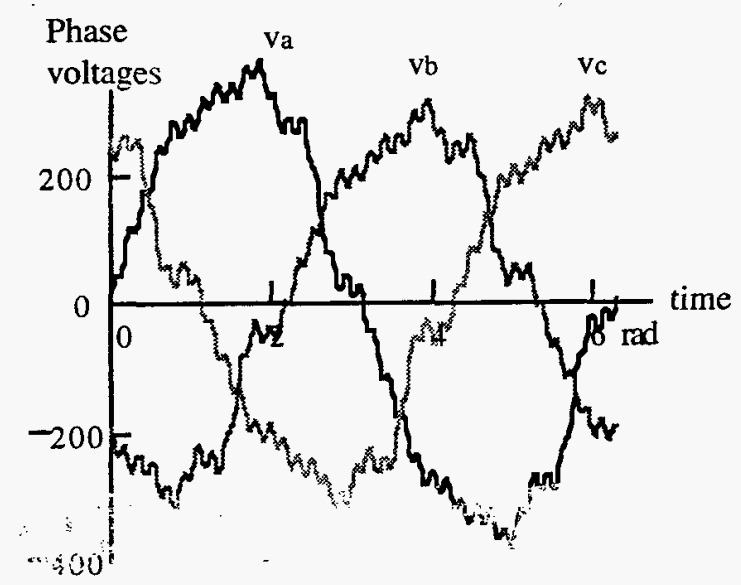

Fig. 1 An example of polluted three-phase voltages

\section{INSTANTANEOUS PHASORS}

\section{Instantaneous Phasors of Balanced Fundamental-} Frequency Voltages or Currents

It is known that a sinusoidal voltage or current can be represented by a phasor that has an rms amplitude and rotates at its angular frequency speed. The phasor's projection to the axis multiplied by $\sqrt{2}$ is the instantaneous value of the voltage or current that the phasor represents. For instance, if $v_{a}, v_{b}$, and $v_{c}$ are instantaneous phase voltages of a balanced fundamentalfrequency supply, they can be obtained from the projections presented by the sine terms in (1),

$$
\begin{aligned}
& v_{a}=V_{\max } \sin \left(2 \pi f t+\vartheta_{0}\right), \\
& v_{b}=V_{\max } \sin \left(2 \pi f t+\vartheta_{0}-2 \pi / 3\right), \quad \text { and } \\
& v_{c}=V_{\max } \sin \left(2 \pi f t+\vartheta_{0}-4 \pi / 3\right),
\end{aligned}
$$

where $V_{\max }=\sqrt{2} \cdot(r m s$ voltage $)$. The $\sqrt{2}$ has been taken care of by using the peak magnitude, $V_{\max }$. The fundamental frequency is denoted by $f$ in $\mathrm{Hz}, \mathrm{t}$ is time in seconds, and $\vartheta_{0}$ is the initial angle.

If the voltages are not balanced, the zero-sequence component for the three-phase voltages is

$$
v_{0}=\frac{1}{3}\left(v_{a}+v_{b}+v_{c}\right)
$$

The zero-sequence power is not a favorable component of the three-phase power supply. It adds unbalance to three phases. However, for balanced threephase voltages or currents the zero-sequence quantity is zero.

The instantaneous phasors' imaginary portion denoted as $v_{a q}, v_{b q}$, and $v_{c q}$ can be derived in a manner similar to (1) but with cosine terms for the orthogonal projections. The result yields

$$
\begin{aligned}
& v_{a q}=\frac{v_{b}-v_{c}}{\sqrt{3}}, \\
& v_{b q}=\frac{v_{c}-v_{a}}{\sqrt{3}}, \quad \text { and } \\
& v_{c q}=\frac{v_{a}-v_{b}}{\sqrt{3}} .
\end{aligned}
$$

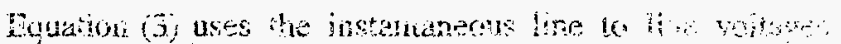
? : se ruaficecd oy zero soguence comproni.

The instantaneous phasor can be expressed in either vector or complex-number format. It is arbitrarily 
chosen to be expressed in a complex-number format in this paper. The instantaneous phasor consists of a real portion that is the difference of (1) and the zero-sequence value of (2) as well as an imaginary portion from (3). Instantaneous values are used instead of rms values.

Equations (1) and (3) are derived on the basis of the assumption that instantaneous phasors of three phases are 120-degree apart from each other. Therefore, the instantaneous phasors given in (4) are 120 degrees apart from each other.

$$
\begin{aligned}
& V_{a}=\left(v_{a}-v_{0}\right)+j v_{a q}, \\
& V_{b}=\left(v_{b}-v_{0}\right)+j v_{b q}, \text { and } \\
& V_{c}=\left(v_{c}-v_{0}\right)+j v_{c q} .
\end{aligned}
$$

The instantaneous phasor magnitude, $V$, of $V_{a}, V_{b}$, and $V_{c}$ can be derived from (4), (3), and (2). The result is that

$$
\left\|V_{a}\right\|=\left\|V_{b}\right\|=\left\|V_{c}\right\|=V
$$

where

$$
V=\sqrt{\frac{2}{9}\left[\left(v_{a}-v_{b}\right)^{2}+\left(v_{b}-v_{c}\right)^{2}+\left(v_{c}-v_{a}\right)^{2}\right]} .
$$

The meaning of $V$ can be seen by rearranging equation (6)

$$
V=\frac{\sqrt{2}}{\sqrt{3}} \sqrt{\frac{\left[\left(v_{a}-v_{b}\right)^{2}+\left(v_{b}-v_{c}\right)^{2}+\left(v_{c}-v_{a}\right)^{2}\right]}{3}} .
$$

It is the equivalent phase magnitude of the three-phase lineto-line voltages averaged in an rms manner.

\section{Instantaneous Phasors of Unbalanced Voltages} Currents

The instantaneous phasors obtained from Equations (2) to (7) can also be used for the polluted threephase voltages or currents that contain harmonics and unbalance. For instance, trajectory of instantaneous phasor $V_{a}$ of (4) for the sample voltages given in Fig. 1 is shown in Fig. 2, where the horizontal axis is for the real portion and the vertical axis is for the imaginary portion (or $\mathrm{x}$ and $y$ axes if one prefers not to use imaginary numbers).

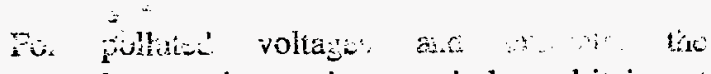

instantaneous phasor trajectory is not a circle, and it is not rotating at a constant speed because of harmonics content and unbalance. The trajectories of phase $b$ and $c$ are the same as phase a except that they are shifted 120 and 240 degrees, respectively. Fig. 3 shows the less complicated shapes of instantaneous phasor trajectories of three phases that contain a half negative-sequence component of the full positive-sequence component.

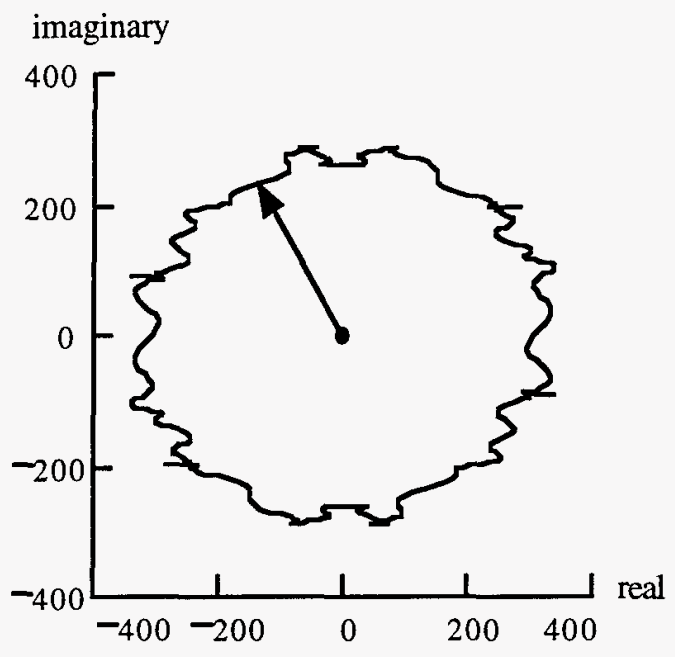

Fig. 2 Trajectory of instantaneous phasor of phase-a voltage given in Fig. 1

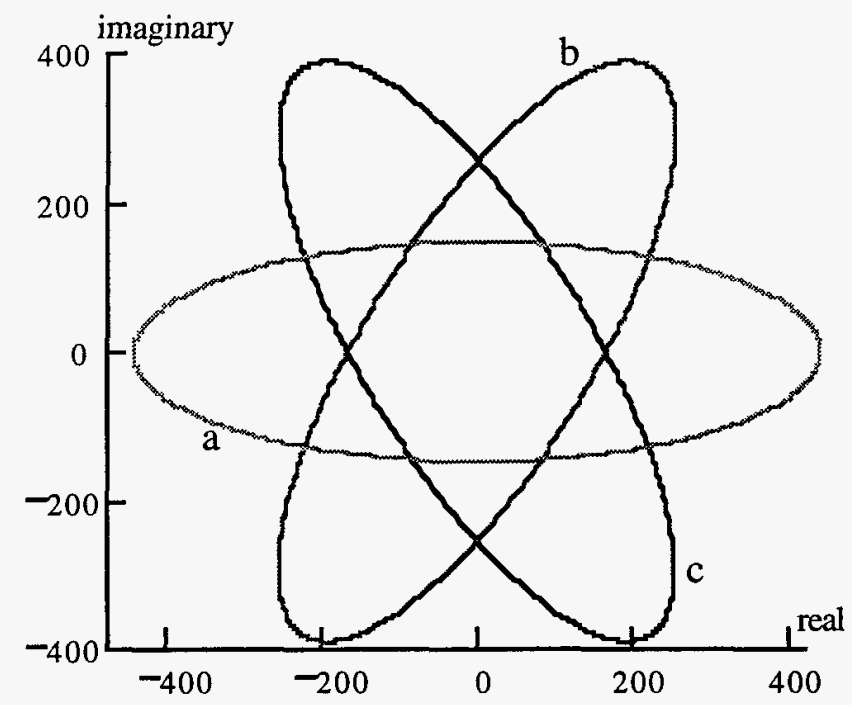

Fig. 3 Trajectories of instantaneous phasors of three phases that contain a half negative-sequence component of the full positive-sequence component.

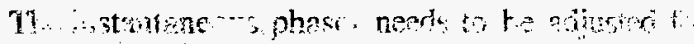

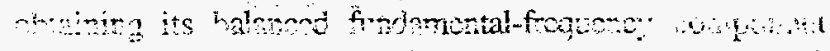
The adjusted phasor should have an average magnitude corresponding to a trajectory of a relatively round circle depending upon the fluctuation of the balanced 
fundamental-frequency component, and the phasor should rotate at a constant fundamental-frequency speed. The following shows a way to use this concept.

\section{OBTAINING INSTANTANEOUS FUNDAMENTAL COMPONENTS THROUGH ADJUSTED INSTANTANEOUS PHASOR}

\section{First cycle (or Start-up period) information}

In order to modify the instantaneous phasor, certain first-cycle (or start-up period, if user requires more than a cycle time) information described in this section is needed. Subsequent instantaneous data readings will instantaneously either modify or retain the first-cycle information.

\subsection{Phase Sequence}

The phase sequence of supply voltages normally would not be changed during a continuous operation. It is obtained from the first cycle and be used throughout the operation. The positive or negative peak magnitudes of each phase voltage are sensed. If the sequence is a-b-c, or $\mathrm{b}-\mathrm{c}-\mathrm{a}$, or $\mathrm{c}-\mathrm{a}-\mathrm{b}$ the sign $=1$, otherwise sign $=-1$.

\subsection{Instantaneous Average Magnitude of Phasor}

The present instantaneous phasor magnitude is used to adjust the past-cycle average phasor magnitudes so that the average phasor magnitude reflects the immediate change of the fundamental-frequency component. The harmonic effect is greatly suppressed by the continuously averaging process.

After passing the first cycle, whenever a new instantaneous phasor is acquired the corresponding phasor data of a cycle earlier is dropped. Let $N$ be the number of data taken in a cycle, $n$ is the sequence of data, and $V_{n}$ is the $n$th value of $V$ defined in (6).

When $1 \leq n \leq N$

$$
\begin{aligned}
& V_{\text {ave }, 0}=0 \\
& V_{\text {ave }, n}=\frac{V_{n}}{n}+V_{\text {ave, } n-1} \frac{n-1}{n} .
\end{aligned}
$$

Subsequently, when $n \geq N+1$

$$
V_{a v e, n}=\frac{V_{n}}{N}+V_{a v e, n-1}-\frac{V_{n-N}}{N}
$$

where the sequence number, $n$, always renews itself to be

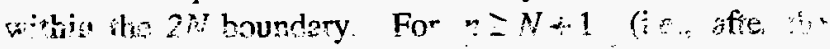

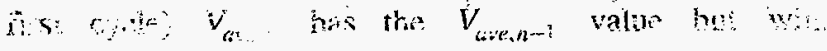
adjustment by discarding the oldest data, $V_{n-N}$, and adding the latest influence of $V_{n}$. Therefore, the instantaneous average phasor magnitude, $V_{a v e, n}$, represents the latest equivalent phase magnitude of three-phase line-to-line voltages averaged in an rms manner.

$$
V_{\text {ave }}=V_{\text {ave, } n} \text {. }
$$

Fig. 4 shows the instantaneous phasor magnitude versus time given in radians for the voltages shown in Fig. 1. The instantaneous average value becomes less fluctuating after the first cycle (i.e., $2 \pi \mathrm{rad}$ ).

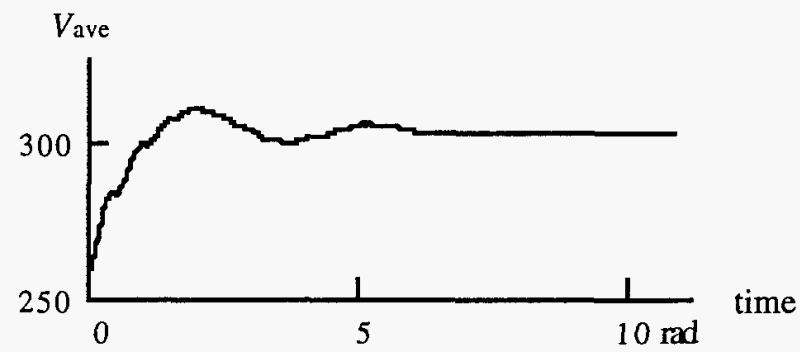

Fig. 4 Instantaneous average phasor magnitude, $V_{a v e}$, versus time given in radians.

In order to minimize data storage size, one possible way is to use a Multiple Access Circular Queue (MACQ) for data flow. A MACQ [14] is a fixed length data structure that preserves order. The source process places information into the MACQ. Once initialized, the MACQ is always full. The oldest data is discarded when the newest data is put into it.

\section{Adjusted Instantaneous Phasors of Unbalanced Voltages or Currents}

The instantaneous phasors of unbalanced voltages given in (4) are adjusted to give balanced phasors.

$$
\begin{aligned}
& V_{a \text { adj }}=V_{a} \frac{V_{a v e}}{V}, \\
& V_{b a d j}=V_{b} \frac{V_{a v e}}{V}, \text { and } \\
& V_{c a d j}=V_{c} \frac{V_{a v e}}{V} .
\end{aligned}
$$

The trajectory of adjusted instantaneous phasor, $V_{a \text { adj }}$, corresponding to phase-a voltage, $v_{a}$, shown in Fig. 1 is illustrated in Fig. 5. The nearly circular-shaped trajectory reveals that the adjusted magnitude of the

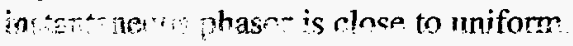

the teal value projection of the adjusted instantaneous phasor, $V_{a \text { adj }}$, is shown in Fig. 6. The nonsinusoidal trace is caused by the non-uniformly rotating 
phasor. If the voltage or current contains harmonics, it is possible that the trace may oscillate such as around the vicinity of each fundamental zero crossing point illustrated in Fig. 1 and Fig. 6. For situations with relatively high content of high-frequency harmonics, a simple low-pass filter that has very little effect on the fundamental frequency component may help to prevent the high-frequency oscillation signals.

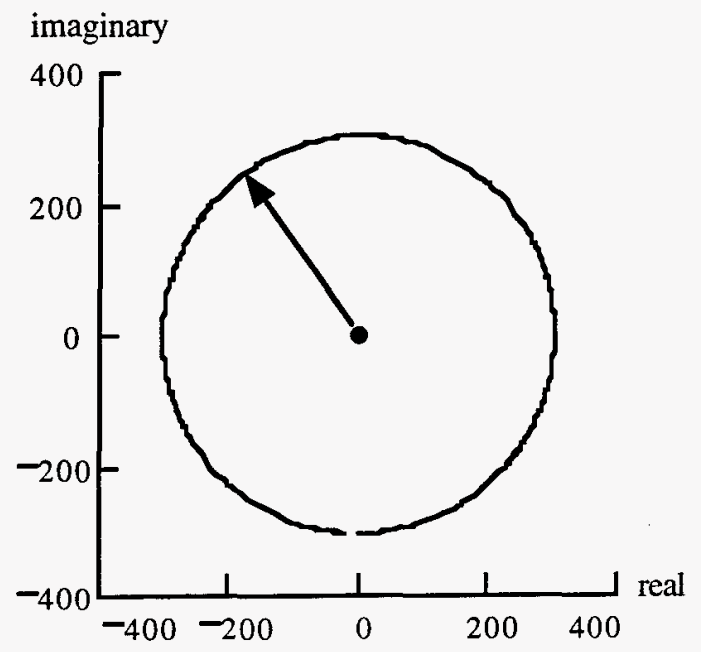

Fig. 5 Trajectory of adjusted instantaneous phasor $V_{a \text { adj }}$

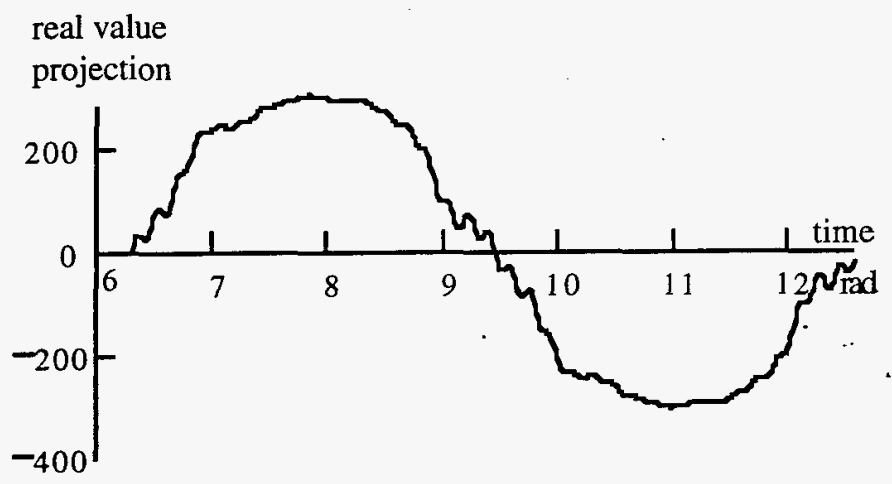

Fig. 6 Real value projection of instantaneous phasor $V_{a \text { adj }}$

\section{Instantaneous Balanced Three-Phase Fundamental Components}

After we have obtained the balanced magnitude of the adjusted phasors, we need to adjust the rotating speed of the phasors. Fig. 7 shows the constant phasor fractional chenge ox a ster corespending to a phesc tho is

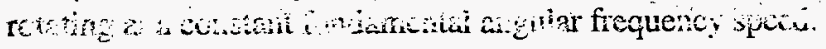

$$
\begin{aligned}
& V_{a \text { adj } n}=V_{a \text { adj } n-1}\left(1+\frac{2 \pi}{N} e^{j \frac{\pi}{2}\left(1+\frac{2}{N}\right) s i g n}\right), \\
& V_{b a d j n}=V_{b a d j n-1}\left(1+\frac{2 \pi}{N} e^{j \frac{\pi}{2}\left(1+\frac{2}{N}\right) s i g n}\right), \text { and } \\
& V_{c \text { adj } n}=V_{\text {cadj } n-1}\left(1+\frac{2 \pi}{N} e^{j \frac{\pi}{2}\left(1+\frac{2}{N}\right) s i g n}\right),
\end{aligned}
$$

where the term sign equals either 1 or -1 that is detected in the first cycle. It dictates the sequence of the instantaneous balanced fundamental-frequency components.

The term $\frac{2 \pi}{N} e^{j \frac{\pi}{2}\left(1+\frac{2}{N}\right) s i g n}$ is the fractional change between the newly defined new and old instantaneous phasors, such as $V_{a \text { adj } n}$ and $V_{a \text { adj } n-1}$ of phase a. $N$ is the number of data readings per cycle, and $n$ is the data reading sequential number. The fractional change can be simplified to be a real constant and an imaginary constant. For example, if $N=360$ the fractional change is as simple as $-1.52 \cdot 10^{-4}+0.017 j$. Fig. 7 shows that the increment, $V_{a \text { adj } n-1} \cdot$ (fractional chnage), can be understood from the relationship between the phasor as a radius and the increment that is closely equal to a section of periphery. The sum of $\pi$ of the interior angles of a triangle is also used in the derivation. The angle between the old phasor and the increment is $\frac{\pi}{2}\left(1+\frac{2}{N}\right) s i g n$.

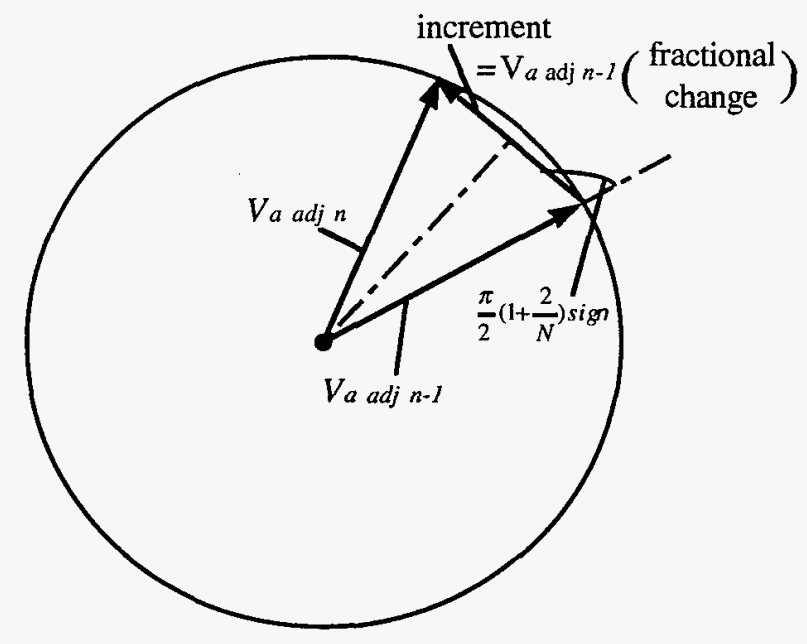

Fig. 7 Phasor increment of a step

The frot phase right afts the first cycle is arbitrarily used as the initial phasor in (11) for this example. Subsequent papers will present the additional rules for selection of the initial and synchronization phasors for severely polluted situations. 
IV. COMPARISON OF RESULTS OBTAINED THROUGH INSTANTANEOUS PHASOR METHOD

\section{AND FOURIER PLUS SYMMETRICAL COMPONENT} METHOD

Fig. 8 shows the comparison of balanced fundamental-frequency components of three phases obtained from the new instantaneous phasor method versus the positive sequence component obtained from the traditional Fourier analysis plus symmetrical component method. Neither the raw data used in this example are filtered nor the initial phasor for (11) is regulated. The discrepancy of magnitude comes from the difference between the balanced fundamental-frequency equivalent phase value of three-phase line-to-line voltages averaged in an rms manner and the positive-sequence component. The magnitude ratio is 1.005. The small discrepancy of phase angle comes from the arbitrarily chosen initial phasor.

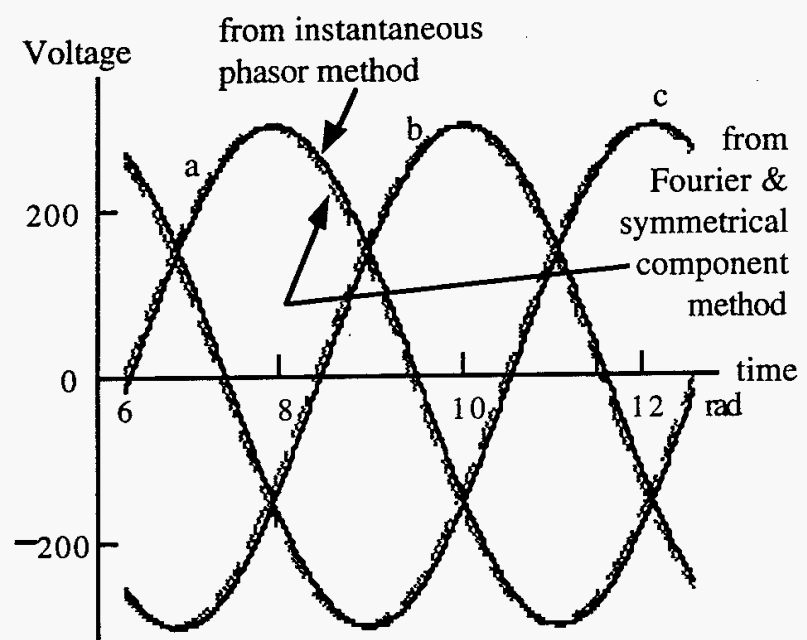

Fig. 8 Balanced fundamental-frequency components obtained from instantaneous phasor method and the traditional Fourier analysis plus symmetrical component method.

The discrepancy shown in Fig. 8 is so small that further effort to correct it for most practical situations similar to the case shown in Fig. 1 is unnecessary. However, it should be pointed out that additional rules would be required for the selection of the initial and synchronization phasors used in (11) for severely polluted situations. These rules will be discussed in subsequent nin.

Comparison between actual phase voltages and instantaneous balanced fundamental-frequency voltages obtained from the instantaneous phasor method is shown in Fig. 9 for the example of Fig. 1.
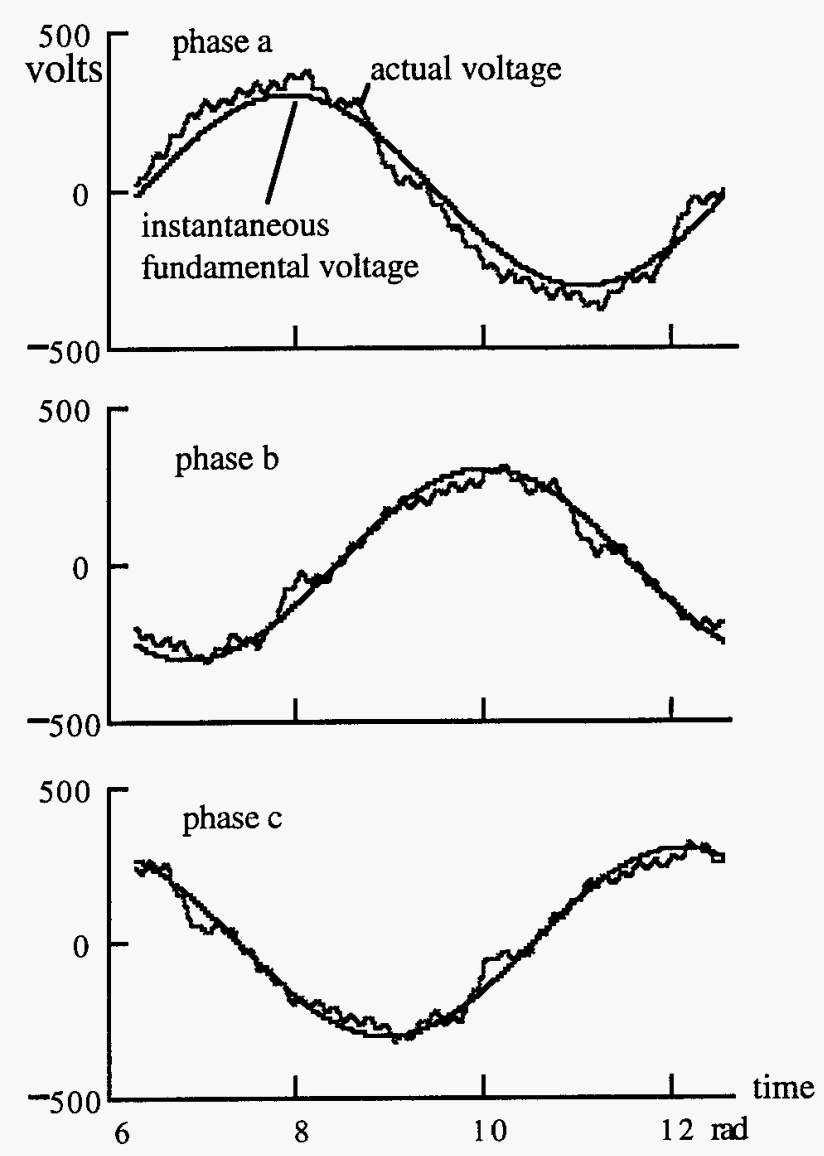

Fig. 9 Comparison between actual phase voltages and instantaneous balanced fundamental-frequency voltages obtained from the instantaneous phasor method

\section{CONCLUSIONS}

A new and simple instantaneous phasor method to obtain instantaneous balanced fundamental-frequency of three-phase voltages or currents is introduced. The potential applications of this technique are for power quality control and for continuous diagnostics.

The definition of instantaneous phasor is established first. The instantaneous phasor's magnitude is adjusted to be the instantaneous average phasor magnitude. Phase sequence is obtained from the first cycle. The

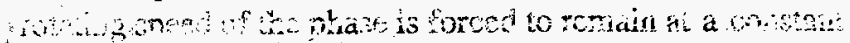

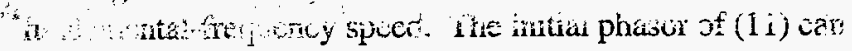
be arbitrarily chosen for most practical cases with a negligible discrepancy. However, rules that will be published in subsequent papers for selection of the initial 
and synchronization phasors have to be used for situations with severe unbalance and extremely high harmonics content.

\section{ACKNOWLEDGMENT}

The author appreciates the financial support provided by DOE's PEBB program. Encouragement from the Power Electronics Group headed by Mr. Donald Adams is gratefully acknowledged.

\section{REFERENCES}

[1] Richard Redl, Paolo Tenti, J. Daan van Wyk, "Power Electronics' Polluting Effects," Spectrum, May 1997, IEEE.

[2] "Power Quality in Commercial Buildings," BR105018, 1995, Electric Power Research Institute, (EPRI), Palo Alto, California.

[3] H. Akagi, Y. Kanazawa, A. Nabae, "Instantaneous Reactive Power Compensators Comprising Switching Devices without Energy Storage Components," IEEE Trans. Ind. Appl., Vol. 20, pp. 625-630, May/June 1984.

[4] L. S. Czarnecki, "Orthogonal Decomposition of the Currents in a 3-Phase Nonlinear Asymmetrical Circuit with a Nonsinusoidal Voltage Source," IEEE Trans. Instrum. Meas., Vol. 37, No. 1, March 1988.

[5] A. Ferrero and G. Superti-Furga, "A New Approach to the Definition of Power Components in Three-Phase Systems Under Nonsinusoidal Conditions," IEEE Trans. Instrum. Meas., Vol. 40, No. 3, June 1991.

[6] J. L. Willems, "A New Interpretation of the AkagiNabae Power Components of Nonsinusoidal Three-Phase Situations," IEEE Trans. Instrum. Meas., Vol. 41, No. 4, August 1992.

[7] L. Rossetto and P. Tenti, "Evaluation of Instantaneous Power Terms in Multi-Phase Systems: Techniques and Application to Power Conditioning Equipment," ETEP Vol.4, No. 6, November/December 1994.

[8] A. Nabae and T. Tanaka, "A New Definition of Instantaneous Active-Reactive Current and Power Based on Instantaneous Space Vectors on Polar Coordinates in

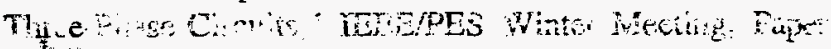
Fr.

[9] F. Z. Peng and J. S. Lai, "Reactive Power and Harmonic Compensation Based on the Generalized
Instantaneous Reactive Power Theory for Three-Phase Power Systems," Proceedings of the 7th International Conference on Harmonics and Quality of Power, pp. 8389, Las Vegas, NV, October 16-18, 1996.

[10] J. S. Hsu, J. D. Kueck, M. OLszewski, D. A. Casada, P. J. Otaduy, L. M. Tolbert, "Comparison of Induction Motor Field Efficiency Evaluation Methods," Paper No. EMC-1-6-6, IEEE/IAS 31st Annual Meeting, October 510, 1996, San Diego, California.

[11] John S. Hsu, Patrick L. Sorenson, "Field Assessment of Induction Motor Efficiency through Air-Gap Torque," paper No. 96WM-130-5EC, presented at the IEEE/PES Winter Meeting, January 21-25, 1996, Baltimore, MD, and accepted for publication in IEEE Transactions on Energy Conversion.

[12] Hsu, John S., "Monitoring of Defects in Induction Motors through Air-Gap Torque Observation," IEEE Transactions on Industry Applications, September 1995, Vol. 31, No. 5, ITIACR, (ISSN 0093-9994), pp. 1016-21.

[13] W. V. Lyon, Transient Analysis of Alternating Current Machinery: An Application of the Method of Symmetrical Components, Wiley, New York, N. Y., 1954.

[14] Dr. Valvano, Microprocessor Application and Organization, Lecture Notes, Electrical and Computer Engineering, College of Engineering, The University of Texas at Austin, Fall 1992.

Dr. John S. Hsu (M 61, SM 89) worked with the Emerson Electric Company, Westinghouse Electric Corporation, and later with the University of Texas at Austin. He is currently a Senior Staff Engineer at the Oak Ridge National Laboratory. He has published nearly forty refereed papers and over one hundred technical publications. $\mathrm{He}$ holds eight patents in rotating machines and power electronics.

* $\quad$ Prepared by the Oak Ridge National Laboratory, Oak Ridge, Tennessee 37831, managed by Lockheed Martin Research Corp. for the U. S. Department of Energy under contract DE-AC05-96OR22464.

The submitted manuscript has been authored by a contractor of the U. S. Government under contract No. DEAC05-96OR22464. Accordingly, the U. S. Government

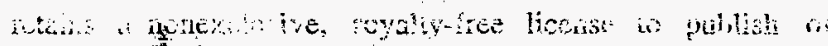

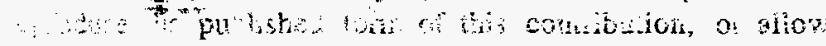
others to do so, for U. S. Government purposes. 
M98001910

|||||||||||||||||||||||||||||||||||||||||||

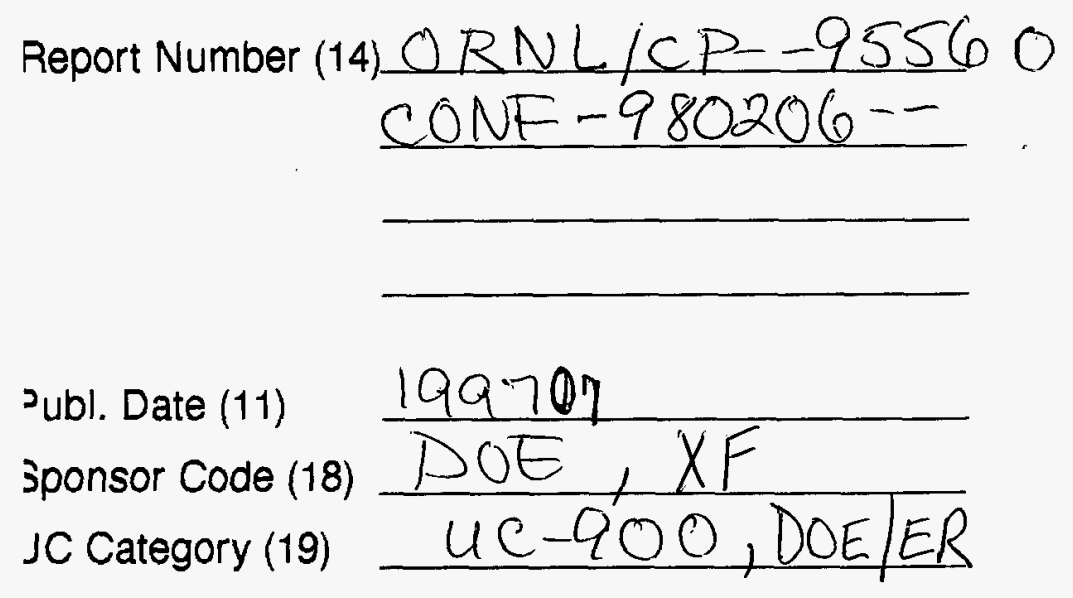

